\title{
THE LOCAL FINITE-AREA PRINCIPLE IN THE HALF-PLANE ${ }^{1}$
}

\section{DANIEL WATERMAN}

1. The familiar finite-area principle of Fejerr asserts that if the image of $|z|<1$ under the analytic mapping $w=f(z)=\sum_{0}^{\infty} a_{n} z^{n}$ is of finite area (counting multiplicities), then $\sum a_{n} z^{n}$ converges a.e. on $|z|=1$ and uniformly on closed arcs of continuity. This result was localized by Zygmund [3] and by Lusin [1] who considered the image of a region bounded by a simple Jordan arc in $|z|<1$ and an $\operatorname{arc} \alpha \leqq \theta \leqq \beta$ of $|z|=1$. They showed that if $a_{n}=o(1)$ then the conclusions of the Fejér theorem hold relative to the arc $[\alpha, \beta]$ and, for $a_{n}=o\left(n^{k}\right), k>-1$, convergence can be replaced by $(C, k)$ summability. It should be noted that the Tauberian conditions in this result are necessary in order that there be a point of convergence (or $(C, k)$ summability) on $|z|=1$.

The result we will establish is a localized finite area theorem for functions analytic in a half-plane.

Theorem. Let $f(s)=\int_{0}^{\infty} e^{-s x} d \gamma(x)$, where $s=\sigma+i \tau$, be analytic in the half-plane $\sigma>0$. Suppose that

$$
\alpha(x)=\sup _{0 \leqq h \leqq 1}|\gamma(x+h)-\gamma(x)|=o(1) .
$$

Let $\Omega$ be a region in $\sigma>0$ bounded by a segment $[i \alpha, i \beta]$ of $\sigma=0$ and $a$ Jordan arc. If

$$
\int_{\Omega} \int\left|f^{\prime}\right|^{2} d \sigma d \tau<\infty
$$

Then $\int_{0}^{\infty} e^{-s x} d \gamma(x)$ converges a.e. on the segment $(i \alpha, i \beta)$ and uniformly on any closed subsegment of continuity. If $\left(\mathrm{A}_{0}\right)$ is replaced by

$$
\alpha(x)=o\left(x^{k}\right), \quad k>0,
$$

then convergence is replaced by $(C, k)$ summability in the conclusion.

It should be noted that $\left(A_{0}\right)$ is a necessary condition for convergence of the integral at one point of $\sigma=0$, but $\left(\mathrm{A}_{k}\right)$, contrary to $[5$, p. 335] is not necessary for $(C, k)$ summability. A counterexample is given in $\$ 3$.

Received by the editors August 18, 1965.

1 This research was supported by National Science Foundation Grants G24841 and GP3987. 
2. We turn now to the proof of the theorem. We consider only the case of $(C, k)$ summability. Clearly there will be no loss of generality if we assume that $\gamma(x) \equiv 0$ for $0 \leqq x<\delta$ and that $0<\alpha<\beta<2 \pi$.

Let $\gamma_{1}$ and $\gamma_{2}$ be the odd and even extensions of $R(\gamma)$ and $g(\gamma)$ to $(-\infty, \infty)$. Set $\phi=\left(\gamma_{1}+i \gamma_{2}\right) / 2$ and $t=-\tau$. Proceeding formally we have

$$
\int_{0}^{\infty} e^{-i \tau x} d \gamma(x)=\int_{-\infty}^{\infty} e^{i t x} d \phi(x)+i \int_{-\infty}^{\infty} e^{i t x}(-i \operatorname{sign} x) d \phi(x) .
$$

Clearly $\phi$ satisfies condition $\left(\mathrm{A}_{k}\right)$ and, by an integration by parts, it may be seen that

$$
\psi(x)=\int_{0}^{x} y d \phi(y)
$$

satisfies condition $\left(\mathrm{A}_{k+1}\right)$.

Let $h$ and $p$ denote positive in tegers which will be chosen as large as is needed. We define

$$
\Phi_{h}(x)=\int_{-\infty}^{x}(i y)^{-h} d \phi(y), \quad \Psi_{h}(x)=\int_{-\infty}^{x}(i y)^{-h} d \psi(y)
$$

and

$$
F(t)=\int_{-\infty}^{\infty} e^{i t x} d \Phi_{h-1}(x), \quad F^{*}(t)=\int_{-\infty}^{\infty} e^{i t x} d \Psi_{h}(x) .
$$

Clearly $\Psi_{h}=-i \Phi_{h-1}$ and so $F^{*}(t)=-i F(t)$.

Let $\lambda(t)$ be a function of period $2 \pi$ and in class $C^{p}$ such that

$$
\begin{aligned}
\lambda(t) & =1 \quad \text { for } \alpha \leqq t \leqq \beta, \\
& =0 \quad \text { for } 0<t<a<\alpha \text { and } \beta<b<t<2 \pi .
\end{aligned}
$$

Let us now consider the formal $h$ th derivative of the Fourier series of $F^{*} \lambda, \mathcal{S}^{(h)}\left(F^{*} \lambda\right)=\sum_{-\infty}^{\infty} \beta_{n} e^{i n t}$ with $\beta_{n}=o\left(n^{k+1}\right)$. Clearly $\mathcal{S}(F \lambda)$ $=-i s\left(F^{*} \lambda\right)$ and so

$$
\mathcal{S}^{(h-1)}(F \lambda)=\sum_{-\infty}^{\infty} b_{n} e^{i n t}
$$

with $b_{n}=o\left(n^{k}\right)$ and $\beta_{n}=-n b_{n}$. Let

$$
g(z)=\sum_{-\infty}^{\infty} b_{n} r^{|n|} e^{i n t}+i \sum_{-\infty}^{\infty}(-i \operatorname{sign} n) b_{n} r|n| e^{i n t}=\sum_{0}^{\infty} c_{n} z^{n}
$$

where $c_{n}=2 b_{n}, z=r e^{i t}$. Then 


$$
\sum_{-\infty}^{\infty} n b_{n} r^{|n|} e^{i n t}+i \sum_{-\infty}^{\infty}(-i \operatorname{sign} n) n b_{n} r^{|n|} e^{i n t}=\sum_{0}^{\infty} 2 b_{n} n z^{n}=z g^{\prime}(z) .
$$

Applying the method employed by Zygmund in [4, Theorem 9] to the function $\psi$ we see that, as $\omega \rightarrow \infty$, the differences

$$
\begin{aligned}
& \int_{-\omega}^{\omega} x e^{i t x} d \phi(x)-\sum_{|n| \leqq \omega} \beta_{n} e^{i n t} \\
& \int_{-\omega}^{\omega} x e^{i t x}(-i \operatorname{sign} x) d \phi(x)-\sum_{|n| \leqq \omega} \beta_{n}(-i \operatorname{sign} n) e^{i n t}
\end{aligned}
$$

are uniformly $(C, k+1)$ summable in $[\alpha, \beta]$, the first difference to zero and the second to a finite value.

We observe now that

$$
\begin{gathered}
R\left(f^{\prime}(s)\right)=-\int_{-\infty}^{\infty} e^{-\sigma|x|} e^{i t x}|x| d \phi(x) ; \\
g\left(f^{\prime}(s)\right)=\int_{-\infty}^{\infty} e^{-\sigma|x|} e^{i t x} i x d \phi(x) .
\end{gathered}
$$

Since the uniform Cesàro summability implies uniform Abel summability we have

$$
\begin{aligned}
& i g\left(f^{\prime}(s)\right)-\sum_{-\infty}^{\infty} n b_{n} e^{i n t} r^{n} \rightarrow 0, \\
& \quad i R\left(f^{\prime}(s)\right)-\sum_{-\infty}^{\infty} n b_{n}(i \operatorname{sign} n) e^{i n t} r^{|n|} \rightarrow \text { finite value }
\end{aligned}
$$

uniformly on $[\alpha, \beta]$ as $\sigma=-\log r \rightarrow 0+$. Hence for some $\epsilon>0$ there is an $M>0$ such that

$$
\left|f^{\prime}(s)\right|^{2}+M \geqq\left|z g^{\prime}(z)\right|^{2}>1 / 2\left|g^{\prime}(z)\right|^{2}
$$

for $\alpha \leqq t \leqq \beta$ and $0<\sigma=-\log r<\epsilon$.

Thus there exists an $M^{\prime}>0$ and a region $\Omega^{\prime}$ in $|z|<1$ bounded by the $\operatorname{arc} \alpha \leqq t \leqq \beta$ of $|z|=1$ and a simple Jordan arc in $|z|<1$ such that

$$
\int_{\Omega^{\prime}} \int\left|g^{\prime}(z)\right|^{2} r d r d t \leqq M^{\prime}+2 \int_{\Omega} \int\left|f^{\prime}(s)\right|^{2} d \sigma d \tau<\infty .
$$

Since $c_{n}=o\left(n^{k}\right)$, the localized finite area theorem of Zygmund is applicable. Thus $\sum_{0}^{\infty} b_{n} e^{i n t}$ is $(C, k)$ summable a.e. on $(\alpha, \beta)$ and uniformly on closed subarcs of continuity. This implies the same for $\sum_{-\infty}^{\infty} b_{n} e^{i n t}$ and $\sum_{-\infty}^{\infty}(-i \operatorname{sign} n) b_{n} e^{i n t}$. 
If we now apply the method of Zygmund to the function $\phi$ satisfying condition $\left(\mathrm{A}_{k}\right)$ we find that the differences

$$
\begin{aligned}
& \int_{-\omega}^{\omega} e^{i t x} d \phi(x)-\sum_{|n| \leqq \omega} b_{n} e^{i n t}, \\
& \int_{-\omega}^{\omega} e^{i t x}(i \operatorname{sign} x) d \phi(x)-\sum_{|n| \leqq \omega} b_{n}(i \operatorname{sign} n) e^{i n t}
\end{aligned}
$$

are uniformly summable $(C, k)$ in $[\alpha, \beta]$, the first to zero and the second to a finite value. The summability properties of the integrals are then the same as those of the series, which establishes the theorem.

3. Consider now the function

$$
\begin{aligned}
\gamma(x)=0 & \text { if } n \leqq x \leqq n+1-1 / 2^{n} \\
& =2^{n} \quad \text { if } n+1-1 / 2^{n}<x<n+1
\end{aligned}
$$

for $n=0,1,2, \cdots$. Then

$$
\begin{aligned}
(C, 1) \int_{0}^{\infty} d \gamma(x) & =\lim (1 / \omega) \int_{0}^{\omega} \int_{0}^{u} d \gamma(x) d u \\
& =\lim (1 / \omega) \int_{0}^{\omega} \gamma(u) d u=1
\end{aligned}
$$

since

$$
[\omega] \leqq \int_{0}^{\omega} \gamma(u) d u \leqq[\omega]+1
$$

But

$$
\sup _{0 \leqq h \leqq 1}|\gamma(x+h)-\gamma(x)|=2^{[x]} \neq o(x) .
$$

Hence $\int_{0}^{\infty} e^{-s x} d \gamma(x)$ is $(C, 1)$ summable at $s=0$, but $\gamma(x)$ does not satisfy condition $\left(A_{1}\right)$ contrary to $[5$, p. 335].

\section{REFERENCES}

1. N. Lusin, On the localization of the principle of finite area, Dokl. Akad. Nauk SSSR 56 (1947), 447-450.

2. F. Wolf, Contributions to the theory of summable trigonometric integrals, Univ. California Publ. Math. 1 (1947), 159-227.

3. A. Zygmund, Sur un théorème de M. Fejêr, Bull. Séminaire Math. Univ. Wilno 2 (1939), 3-12.

4. - On trigonometric integrals, Ann of Math. 48 (1947), 393-440.

5. —, Trigonometric series, Vol. II, 2nd ed., Cambridge Univ. Press, New York, 1959.

Wayne State University 